\title{
An optimal gains matrix for time-delay feedback control
}

\author{
James Biggs* Colin R.McInnes ** \\ * Department of Mechanical Engineering, University of Strathclyde, \\ Glasgow,The United Kingdom (e-mail: james.biggs@strath.ac.uk). \\ ** Department of Mechanical Engineering, University of Strathclyde, \\ Glasgow,The United Kingdom (e-mail: colin.mcinnes@strath.ac.uk)
}

\begin{abstract}
In this paper we propose an optimal time-delayed feedback control (TDFC) for tracking unstable periodic orbits (UPOs). It is shown that TDFC will drive a trajectory onto a periodic orbit while minimising an integral of a cost function of the error in periodicity and the control effort. This optimal TDFC relies on the linearisation about the delayed trajectory not the UPO itself and therefore can be implemented without a priori knowledge of a reference orbit. This optimal TDFC is applied to the problem of tracking an unstable periodic orbit in the nonlinear equations describing the circular restricted three-body problem. The results of this investigation demonstrate that TDFC could efficiently drive a spacecraft onto a periodic orbit in the vicinity of a (UPO) halo orbit.
\end{abstract}

\section{INTRODUCTION}

Time-delayed feedback control (TDFC) is an efficient method for stabilising unstable periodic orbits (UPOs) embedded in chaotic attractors. The method of TDFC is often referred to as the method of time-delay autosynchronisation, since the stabilisation of UPOs manifests itself as a synchronisation of the current state of the system with its delayed state. The method of TDFC, as proposed by Pyragas (1992) is a non-invasive method that requires no exact knowledge of the form of the periodic orbit, only the period of the desired orbit. The method is based on applying a simple continuous feedback control that is proportional to the deviation of the current state of the system from its state one period in the past, explicitly:

$$
\boldsymbol{u}(t)=-K(\boldsymbol{X}(t)-\boldsymbol{X}(t-\tau))
$$

where $\boldsymbol{u}(t)$ is the control vector, $K$ is a gains matrix, $\boldsymbol{X}(t)$ is the $n$-dimensional current state vector and $\boldsymbol{X}(t-\tau)$ is the state vector one period in the past with the period $\tau$. From equation (1) it can be seen that if the trajectory lies exactly on the periodic orbit then the control signal vanishes.

The concept of optimal time-delayed feedback control has been proposed previously in the field of controlling chaos by Basso et. al. (1998) and Tian et. al. (1998). In this paper we present an alternative method for computing the optimal gains matrix $K$. Moreover, we yield the necessary conditions for optimality (minimising a function of tracking error and control effort) and present them conveniently as matrix differential Ricatti equations. Before proceeding we emphasise that the proposed optimal TDFC is convenient for experimental situations, since it does not require any reference to the UPO, that is, the computation of the gains matrix is not computed about the unknown UPO, but the delayed trajectory $\boldsymbol{X}(t-\tau)$.
The aim of this paper is to apply the TDFC in equation (1) to a nonlinear system of the form:

$$
\dot{\boldsymbol{X}}(t)=f(\boldsymbol{X}(t))+\boldsymbol{u}(t)
$$

with the aim to optimally track a periodic orbit defined by:

$$
\boldsymbol{X}(t)=\boldsymbol{X}(t-\tau)
$$

$\forall t$ where $\tau$ is the period of the orbit.

In the second part of this paper we apply our optimal TDFC to the problem of driving a spacecraft onto a periodic orbit in the vicinity of an unstable (halo) periodic orbit in the nonlinear equations of the circular restricted three-body problem (CRTBP).

\section{OPTIMAL TRACKING OF UNSTABLE PERIODIC ORBITS}

In this section we show that a TDFC in the form of equation (1) optimally drives a trajectory onto a periodic orbit in the vicinity of an unstable orbit defined by equation (3) with respect to a quadratic cost function of the error in periodicity and control effort. Firstly, we note that as $\boldsymbol{X}(t)$ is a solution of the original system (2) on the interval $t \in[-\infty, \infty]$ with $\boldsymbol{u}(t)=0$ then the delayed trajectory is also a solution of $(2)$ on the interval $t \in[-\infty, \infty]$, namely:

$$
\dot{\boldsymbol{X}}(t-\tau)=f(\boldsymbol{X}(t-\tau)),
$$

it follows that subtracting (4) from (2) yields the error dynamical system:

$$
\dot{e}(t)=F(e(t))+\boldsymbol{u}(t),
$$

where

$$
\boldsymbol{e}(t)=\boldsymbol{X}(t)-\boldsymbol{X}(t-\tau)
$$


where $\boldsymbol{e}(t)$ is referred to as the error in periodicity and

$$
F(\boldsymbol{e}(t))=f(\boldsymbol{X}(t))-f(\boldsymbol{X}(t-\tau)) .
$$

Additionally, taking the Taylor expansion of $f(\boldsymbol{X}(t))$ about the delayed trajectory $\boldsymbol{X}(t-\tau)$ gives:

$$
\begin{aligned}
& f(\boldsymbol{X}(t))=f(\boldsymbol{X}(t-\tau))+A(t)(\boldsymbol{X}(t)-\boldsymbol{X}(t-\tau))+ \\
& \quad+[\text { H.O.T }]
\end{aligned}
$$

where $[H . O . T]$ are higher order terms and

$$
A(t)=\left.\frac{\partial f(\boldsymbol{X}(t))}{\partial \boldsymbol{X}(t)}\right|_{\boldsymbol{X}_{(t-\tau)}}
$$

It follows from substituting equation (6) and (7) into equation (8) that:

$$
F(\boldsymbol{e}(t))=A(t) \boldsymbol{e}(t)+[H . O . T]
$$

and assuming that the initial error in periodicity $\boldsymbol{e}(t)$ is small enough, we may neglect higher order terms in $\boldsymbol{e}(t)$ and therefore equation (5) and equation (10) yield the linear time-varying error dynamical system:

$$
\dot{\boldsymbol{e}}(t)=A(t) \boldsymbol{e}(t)+\boldsymbol{u}(t)
$$

for the controlled system (11) we wish to apply the control (1) to drive $\boldsymbol{e}(t) \rightarrow 0$ while minimising a practically meaningful cost function. We choose to minimise a performance index $J$ which is a quadratic function of the control effort $\boldsymbol{u}(t)$ and error in periodicity $\boldsymbol{e}(t)$ :

$$
J=\frac{1}{2} \int_{0}^{\infty}\left(\boldsymbol{e}^{T}(t) Q \boldsymbol{e}(t)+\boldsymbol{u}^{T}(t) R \boldsymbol{u}(t)\right) \quad d t
$$

where $Q$ and $R$ are real symmetric positive definite matrices. Therefore, it follows from optimal control theory for linear time-varying systems (as described by Lewis (1986) and Barnett (1985)) that the optimal control for the linear time-varying system (11) subject to the cost function (12) is $\boldsymbol{u}(t)=-K \boldsymbol{e}(t)$ which from equation (6) corresponds to a time-delayed feedback control of the form $\boldsymbol{u}(t)=-K(\boldsymbol{X}(t)-\boldsymbol{X}(t-\tau))$ with $K=R^{-1} P(t)$ and where $P(t)$ satisfies the matrix differential Ricatti equations

$$
\dot{P}(t)=P(t) R^{-1} P(t)-A(t)^{T} P(t)-P(t) A(t)-Q
$$

The necessary conditions for optimality (13) can easily be generalised to include the situation where the number of controls $m$ is less than the dimension of the phase space $n$ by replacing equation (11) with the linear time-varying error dynamical system:

$$
\dot{\boldsymbol{e}}(t)=A(t) \boldsymbol{e}(t)+B \boldsymbol{u}(t)
$$

where $B$ is a $n \times m$ matrix. In this case $K=R^{-1} B^{T} P(t)$ where $P(t)$ is a solution of the matrix differential Ricatti equation:

$$
\dot{P}(t)=P(t) B R^{-1} B^{T} P(t)-A(t)^{T} P(t)-P(t) A(t)-Q(15)
$$

To apply this optimal TDFC in practise the differential equation (15) has to be integrated simultaneously with the equations of motion to obtain the instantaneous optimal time-varying gains matrix $K$.

\section{EXAMPLE: TRACKING UNSTABLE PERIODIC ORBITS IN THE RESTRICTED THREE-BODY PROBLEM}

In this section we show by example that the optimal TDFC can be used to drive a spacecraft onto a periodic orbit in the vicinity of an unstable (halo) orbit (halo orbits were described in detail by Szebehely (1967)). Additionally, a number of halo orbits have been identified numerically in the circular restricted three-body problem (CRTBP) by Gomez et. al. (2001).

The CRTBP describes the motion of a small body (spacecraft) under the gravitational influence of two massive bodies rotating in circular orbits around their common centre of mass. It is well known that there are 5 equilibrium points (Libration points) in the rotating coordinate system which correspond to points where the magnitude of the centrifugal acceleration and the acceleration due to the gravitational forces of the Earth and the Sun are equal. Halo orbits represent one type of three-dimensional periodic solution known to exist near the collinear points. These types of periodic arcs are often envisioned as ideal outposts for astronomical observation. For example the international Sun-Earth explorer placed an observatory in a Halo orbit about the $L_{1}$ point, located along the SunEarth line between the Earth and the Sun (as described by Richardson (1980)).

Halo orbits are highly unstable and in such dynamically sensitive regimes stabilisation is required to counteract the effects of numerical error during the integration process, particularly when propagating the path over multiple revolutions. Methods such as LQR control described by Wie (1987) and $H_{\infty}$ control described by Kulkarni et. al. (2006) have previously been used to stabilise spacecraft flight about halo orbits. The present study focuses on the use of the optimal time-delay feedback control described in this paper as a means to autonomously control spacecraft on halo orbits.

Time-delay feedback control has an advantage over the previous methods by Wie (1987) and Kulkarni et. al. (2006) in that it does not require the construction of a reference orbit a priori. Here time-delay feedback control is proposed to enhance the autonomy of spacecrafts operating in the vicinity of libration points. If the spacecraft requires autonomous on-board navigation time-delayed feedback can, in principle, achieve a periodic path autonomously. However, in order to minimize the control effort, the spacecraft's path must evolve in the vicinity of a periodic orbit in phase space. Again, an a priori reference trajectory is not required and therefore the time-delay control will manoeuvre the spacecraft onto a closed path autonomously. The position of the small body (spacecraft) is denoted by $x, y, z$ in astronomical units (AU) and the nonlinear equations of the CRTBP are then given as:

$$
\begin{aligned}
& \ddot{x}-2 \dot{y}=\Omega_{x}+u_{x}, \\
& \ddot{y}+2 \dot{x}=\Omega_{y}+u_{y}, \\
& \ddot{z}=\Omega_{z}+u_{z},
\end{aligned}
$$

where $\Omega_{x}, \Omega_{y}, \Omega_{z}$ are the partial derivatives of $\Omega$ with respect to $x, y, z$ respectively and where 


$$
\begin{aligned}
& \Omega=\frac{1}{2}\left(x^{2}+y^{2}\right)+\frac{1-\mu}{r_{1}}+\frac{\mu}{r_{2}}, \\
& r_{1}^{2}=(x-\mu)^{2}+y^{2}+z^{2}, \\
& r_{2}^{2}=(x-\mu+1)^{2}+y^{2}+z^{2}
\end{aligned}
$$

where a 'dot' denotes differentiation with respect to time, $\mu$ is the mass parameter which for the Earth-Sun system is $\mu=0.000003$ and $\boldsymbol{u}(t)=\left[u_{x}, u_{y}, u_{z}\right]^{T}$ are the orthogonal acceleration controls. These controls realistically reflect the type of continuous control a conventional spacecraft would use to stabilise its motion about a halo orbit. Firstly we expand the state space of the nonlinear equations (16) and write them as a set of first order ordinary differential equations of the form $\dot{\boldsymbol{X}}(t)=f(\boldsymbol{X}(t))+\boldsymbol{u}(t)$. The error dynamical system describing the time evolution of the error in periodicity $\boldsymbol{e}(t)$ can then be expressed explicitly in the form of a linear time-varying system:

$$
\dot{\boldsymbol{e}}(t)=A(t) \boldsymbol{e}(t)+B \boldsymbol{u}(t)
$$

where $A(t)$ is given by:

$A(t)=\left(\begin{array}{ll}0 & I \\ J & \Omega\end{array}\right), \quad J=\left(\begin{array}{lll}a & b & c \\ d & e & f \\ g & h & i\end{array}\right), \quad \Omega=\left(\begin{array}{ccc}0 & -2 & 0 \\ 2 & 0 & 0 \\ 0 & 0 & 0\end{array}\right)$

where

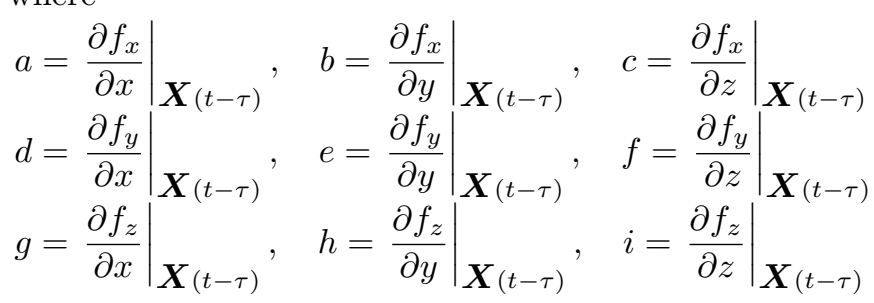

and with

$$
B=\left[\begin{array}{llllll}
0 & 0 & 0 & 0 & 0 & 1 \\
0 & 0 & 0 & 0 & 1 & 0 \\
0 & 0 & 0 & 1 & 0 & 0
\end{array}\right]^{T}
$$

To solve this optimal tracking problem we then have to integrate the differential equation (15) along the delayed trajectory to obtain the gains matrix $K$. This procedure involves determining the most appropriate real symmetric positive definite matrices $Q$ and $R$ that yield the best possible control performance in terms of the cost function (12). The simple and easy to implement procedure used to determine $Q$ and $R$ in this paper is to solve equation (15) for $\dot{P}(t)=0$ at $t=0$ to obtain a constant $K$ matrix for different values of $Q$ and $R$. We then obtain a constant $K$ that gives us the best control performance given these matrices. Having obtained the most appropriate constant $K$ we compute the corresponding $P$ matrix through the equation $P=B R K$. This $P$ will then serve as the initial condition $P(0)$ in the numerical integration of (15) to give us the true optimal gains matrix for the linear time-varying system.

For the case where there is no control $\boldsymbol{u}(t)=0$, initial conditions that yield halo orbits in the Earth-Sun system have been stated numerically by Gomez et. al. (2001). We perturb from these initial conditions to yield an almost (halo) periodic orbit of period $\tau=3.061$. TDFC then drives the spacecraft onto and stabilises about the halo orbit. The controlled trajectory over 10 years is illustrated in Figure 1 (i) and the acceleration controls required to drive the trajectory onto a periodic orbit are illustrated in Figure 1 (ii). In Figure 1 (i) the trajectory converges to a periodic orbit in the vicinity of a halo orbit and is maintained despite the highly unstable nature of a natural halo orbit.

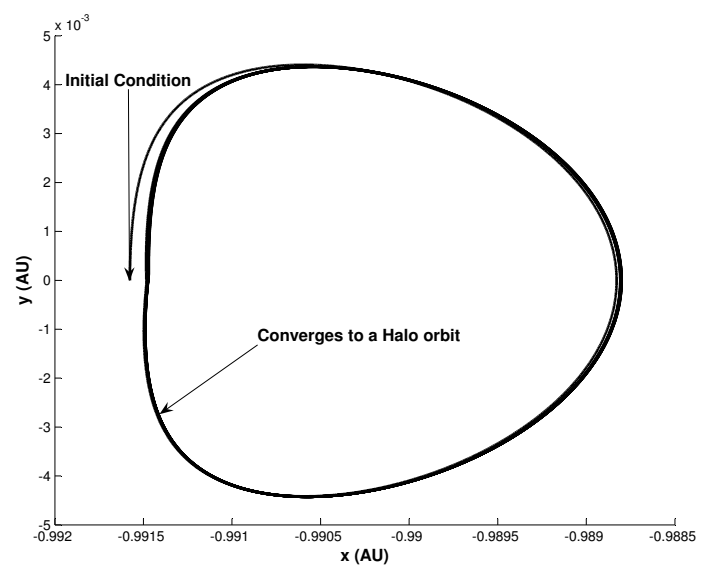

(i)
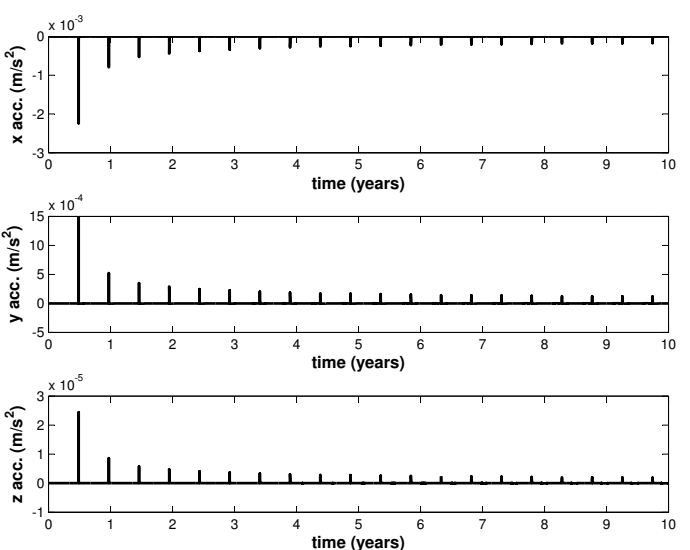

(ii)

Fig. 1. (i) Time-delay control drives the trajectory onto a periodic orbit in the vicinity of a halo orbit (ii) the acceleration controls $\left(\mathrm{m} / \mathrm{s}^{2}\right)$ required to drive the trajectory onto a periodic orbit

The magnitudes of the controls are shown to reduce with time, which through equation (1) implies that TDFC drives the error function to zero. Future work will include a Floquet analysis of the closed loop system to assess the stability properties of the TDFC induced periodic orbit.

\section{CONCLUSIONS}

This paper addresses the problem of driving trajectories of nonlinear systems onto periodic orbits using an optimal time-delayed feedback control (TDFC). We state the necessary conditions for optimality with respect to a cost function of the error in periodicity of the trajectory and the control effort. This setting naturally presents an optimal TDFC which can be implemented without knowledge of a 
reference orbit a priori. This optimal TDFC is then applied to the problem of driving a trajectory onto a periodic orbit in the nonlinear equations describing the circular restricted three-body problem. The results of this investigation demonstrate that TDFC could efficiently drive a spacecraft onto a periodic orbit in the vicinity of a halo orbit.

\section{REFERENCES}

K. Pyragas. Continuous control of chaos by self-controlling feedback. Physics Letters A, 170:421-428, 1992.

M. Basso, L. Genesio, L. Giovanardi, A. Tesi, G. Torrini. On Optimal Stabilization of Periodic Orbits via timedelayed feedback control. International Journal of Bifurcation and Chaos, 8:1699-1706, 1998.

Y-P. Tian, X. Yu. Stabilizing unstable periodic orbits of chaotic systems via an optimal principle. Journal of the Franklin Institute, 337:771-779, 2000.

F. L. Lewis. Optimal Control. New York, Wiley, 1986.

S. Barnett, R. G. Cameron. Introduction to Mathematical Control Theory. Oxford Applied Mathematics and Computing Science Series, 1985.

D. L. Richardson. Halo orbit formulation for the ISEE-3 mission. Journal of Guidance and Control, 3:543-548, 1980.

V. Szebehely. Theory of Orbits: The restricted problem of three bodies. Academic Press, New York, 1967.

G. Gomez, J. Llibre, R. Martinez, C. Simo. Theory of Orbits: The restricted problem of three bodies. Vol. 1 Fundamentals: The Case of Collinear Libration Points. World Scientific, 2001.

B. Wie. Space Vehicle Dynamics and Control. AIAA Education Series, New York, 1987.

J. E. Kulkarni, M. E. Campbell, G. E. Dullerud. Stabilization of Spacecraft Flight in Halo Orbits: An $H_{\infty}$ approach. IEEE Trans. on Control systems Technology, 14:572-578, 2006. 